\title{
Article
}

\section{Injury risk and patterns in newly transferred football players: A case study of 8 seasons from a professional football club}

Carling, C., McCall, A., Le Gall, F., and Dupont, G.

Available at http://clok.uclan.ac.uk/19468/

Carling, C. ORCID: 0000-0002-7456-3493, McCall, A., Le Gall, F., and Dupont, G. (2017) Injury risk and patterns in newly transferred football players: A case study of 8 seasons from a professional football club. Science and Medicine in Football, 2 (1). pp. 47-50. ISSN 2473-3938

It is advisable to refer to the publisher's version if you intend to cite from the work. http://dx.doi.org/10.1080/24733938.2017.1370123

For more information about UCLan's research in this area go to http://www.uclan.ac.uk/researchgroups/ and search for <name of research Group>.

For information about Research generally at UCLan please go to http://www.uclan.ac.uk/research/

All outputs in CLoK are protected by Intellectual Property Rights law, including Copyright law. Copyright, IPR and Moral Rights for the works on this site are retained by the individual authors and/or other copyright owners. Terms and conditions for use of this material are defined in the policies page.

\section{CLoK}

Central Lancashire online Knowledge www.clok.uclan.ac.uk

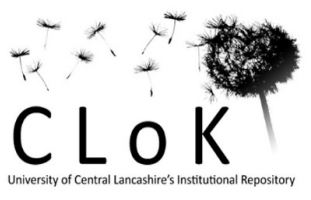


Title: Injury risk and patterns in newly transferred football players: A case study of 8 seasons from a professional football club

Authors: Christopher Carling ${ }^{1}$, Alan $\mathrm{McCall}^{2}$, Franck Le Gall ${ }^{3}$, Gregory Dupont ${ }^{3}$

\section{Institution:}

${ }^{1}$ Institute of Coaching and Performance, University of Central Lancashire, UK

${ }^{2}$ Research Department for Sports and Exercise Science, Napier University, Edinburgh, UK

${ }^{3} \mathrm{LOS}$

\section{Corresponding author:}

Christopher Carling

Correspondence: Institute of Coaching and Performance, University of Central Lancashire, Preston, UK.

Phone: 00.33.6.0392 1863

Email: christopher.carling@gmail.com

Word count: 2264 
Title: Injury risk and patterns in newly transferred football players: A case study of 8 seasons from a professional football club

Running head: Injury in elite football

\begin{abstract}
This case study investigated injury risk and patterns in players newly transferred to a professional football club. Time-loss injuries were recorded prospectively over 8-seasons (2008-2015). Injury incidence overall, in match and training, and patterns (contact, non-contact, sprain, strain, overuse and re-injury) were compared in transferred players $(n=25)$ across their first versus second seasons and with those in players currently at the club at the moment of the transfer ( $\mathrm{n}=55$ individual players, 134 in total). Incidence Rate Ratios [IRR] in transferred players in their first versus second competitive season ranged from a 0.9 lower risk in training to a 1.5 higher risk of sustaining a contact injury (respective $\mathrm{p}$ values: $0.74,0.19$, inferences: unclear, likely harmful) in the first season. IRR for transferred players in their first and second seasons compared to rates in the same seasons in current players ranged from a 0.5 lower risk of incurring an overuse injury to a 1.1 higher risk of match injury (respective p values: $0.18,0.89$, inferences: unclear, possibly harmful), both occurring in season 1. For the between season and group comparisons, effect sizes regarding mean injury layoff time and matches missed ranged from trivial to small (0.03-0.22). Although limited to one club, these findings are positive as generally there was no meaningful increase in injury risk or burden in newly transferred players. Potential explanations include systematic pre-participation screening and injury prevention protocols and player rotation strategies in place at the club.
\end{abstract}

Key terms: injuries, incidence, burden, soccer, player transfers. 


\section{Introduction}

In contemporary elite football, success is heavily reliant upon the individual and collective physical, psychological, tactical and technical skills of players. Effective squad management policies, notably in terms of player selection and rotation in order to reduce the chance of over-exposure and the ensuing risk of under-performance and injury are also vital over the course of the season (Carling McCall, Le Gall, \& Dupont, 2015). Indeed, the ability of players to remain injury-free hence maximising availability to play is strongly related to results and league ranking (Eirale, Tol, Farooq, Smiley, \& Chalabi, 2012; Hägglund, Waldén, Magnusson, Kristenson, Bengtsson et al., 2013).

Success at elite standards also evidently hinges on a club's ability to assemble the 'best' team and this process is strongly dependent on transfer activities (Liu, Liu, Lu, Wang \& Wang, 2016). Yet, to our knowledge, while a large number of potential internal and external risk factors for injury in male elite football players have been examined (Stege, Stubbe, Verhagen, \& van Mechelen, 2011), no study has investigated the risk of injury in newly transferred players. In a related article, players undergoing their first season with a first-team contract after being promoted from an elite club youth academy sustained a greater incidence of stress-related bone injuries than in established first-team players although general injury rates were lower (Kristenson, Waldén, Ekstrand, \& Hägglund, 2013). Several factors were discussed by the authors that could influence injury occurrence in players integrating a new playing environment including adaptation to new training methods and changes in training and match exposure and loads. If newly transferred players cannot play due to injury, the potential repercussions on team performance and the burden notably from a financial perspective particularly following a large transfer fee can be substantial.

The aim of the present case study was to investigate injury risk and patterns in senior players newly transferred to a professional football club.

\section{Methods}

This prospective observational study investigated injuries sustained over an 8-season period in a cohort of male professional football players belonging to the first-team squad of a French Ligue 1 Club (20082015). While all data arose as a contractual condition of employment in which players were routinely monitored over the course of the competitive season (Winter \& Maughan, 2009), approval for the study from the present club was obtained. To ensure confidentiality, all data were anonymised before analysis. The club's medical and physical conditioning staff were unchanged over the entire study span while the coaching staff changed for the final two seasons.

Over the 8-season span, individual exposure time to all club match-play and training sessions was recorded for players belonging to the first-team squad by the club's sports scientist. Only players considered potential first-team starters were included. This categorisation was done following consultation with the club's coaching staff. Players were divided into two cohorts for comparisons: Transferred players versus current (established) players. A total of 25 individual player transfers (age 
$25.8 \pm 3.6$ years on joining the club) occurred with players followed over their first and second seasons in the club (50 player seasons). The cohort of current players who were already playing for the club at the moment the player was transferred included 55 individual players (134 players in total, age $25.1 \pm 4.9$ years, 134 player seasons).There were no minimum inclusion criteria according to the number of minutes played in training or competition.

The injuries sustained in training and match-play were prospectively diagnosed and documented by the same sports physician over the entire study period. The definitions of injury were based on those recommended by International Football Injury Consensus Groups (Fuller et al., 2006; Hagglund, Walden, Bahr, \& Ekstrand, 2005). Injury: time-loss injury resulting from playing football and leading to a player being unable to fully participate in future training or match play irrespective of whether a training session actually took place on the day following injury or the player was selected to play in the next match. A recurrent injury was described as an injury of the same type and at the same site as an index injury and that occurred within 2 months after a player's return to full participation from the index injury. Information on the cause of injuries (non-contact or contact) was collected via direct questioning of the player by the club physician. If there was cause for doubt on the cause of an injury then this was classified as 'unknown'. The layoff time of the injury was determined according to the number of days the player was absent from and unable to take full part in training or competition. The number of matches missed over the course of each injury was recorded.

Due to its case study nature, explorative statistics were employed. Standard procedures were used to calculate frequencies, means and standard deviations. The incidence of injury (number of injuries per 1000hours exposure to play) and incidence rate ratios (IRR) for comparisons across cohorts are reported. Both are presented with 95\% Confidence Intervals (95\%CI). IRR were tested for significance using derived Z statistics (Hayen \& Finch, 2010). Given the real-world nature of the study, magnitude-based inferences were also used to determine any practically relevant differences in injury rates between groups (Batterham \& Hopkins, 2006; Hopkins, 2010). Thresholds for beneficial and harmful effects were 0.90 and 1.11 respectively. Likelihoods were subsequently generated and thresholds for assigning qualitative terms to chances were assigned as follows (Hopkins, 2006): <1\%, almost certainly not; <5\%, very unlikely; $<25 \%$, unlikely; $<50 \%$, possibly not; $\geq 50 \%$, possibly; $\geq 75 \%$, likely; $\geq 95 \%$, very likely; $\geq 99 \%$, almost certainly. The magnitudes of differences between groups were considered practically meaningful when the likelihood was $>75 \%$. All statistical procedures were performed using Excel (Microsoft, Richmond, USA).

Player exposure time, mean layoff time for injuries and mean number of matches missed were compared across cohorts using Effect Sizes (ES). The magnitude of the ES differences was interpreted as trivial $(<0.2)$, small $(>0.2-0.6)$, moderate $(>0.6-1.2)$, large $(>1.2-2.0)$ and very large $(>2.0-4.0)$ (Batterham \& Hopkins, 2006).

\section{Results}


The overall exposure time to play for transferred and current players is reported in Table 1. Exposure time for transferred players in their first and following seasons equated to $315 \pm 89$ and $344 \pm 72$ hours of play per player respectively while a value of $347 \pm 61$ hours was observed per player in those who were currently members of the squad (ES range: 0.05-0.48, trivial to small differences).

Altogether, 68 and 64 injuries $(2.7 \pm 2.2$ and $2.6 \pm 2.3$ per player) were sustained in transferred players in their first and following seasons respectively while current players incurred a total of 422 injuries (3.1 \pm 8.3 per player). Incidences for injury overall and in match and training, and injuries classed as contact, noncontact sprains, strains, overuse and re-injuries did not differ significantly in transferred players in in their first versus following seasons (Table 2). Higher incidences of injury overall, in matchplay and injuries classed as contact were nevertheless observed in the transferred player's first season with the greater risk rates respectively classed as possibly harmful for the two former categories (IRR both 1.2) and likely harmful for the latter (IRR: 1.5). No significant differences were observed for incidences of the aforementioned variables in transferred players in their first versus following seasons compared to those observed during the same seasons in current members of the squad. In transferred players however, lower injury rates were notably observed overall in their first season (IRR: 0.8, possibly beneficial), in training in both seasons (IRR: both 0.7, likely beneficial) and for contact type injuries in their second season (IRR: 0.7, likely beneficial) while match injury rates incurred in their first season were higher (IRR: 1.1, possibly harmful).

Comparison of the mean layoff time per injury between groups reported trivial effect size differences (ES range: 0.03-0.09): $12.1 \pm 27.4$ and 10.1 \pm 15.4 days per injury in the first and following seasons for transferred players compared to $10.7 \pm 18.3$ days for current players. The mean number of matches missed was $5.4 \pm 8.1$ and $3.8 \pm 6.0$ in transferred players in their first and following seasons compared to $5.3 \pm 7.5$ in current players (ES range: $0.01-0.22$, trivial to small).

\section{Discussion}

The present case study investigated injury rates and patterns in players newly transferred to a professional football club. Findings generally showed no differences in the risk and patterns of injury observed in newly transferred players' in their first compared to their following seasons although a practically meaningful higher incidence of contact-type injuries was observed in the former. Similarly, no differences were observed in transferred players in either their first or following seasons compared to results in the same seasons in current members of the squad although practically meaningful lower values for overall, training and contact-type injury rates were observed in the transferred players.

Injury rates were compared across the first and second seasons in newly transferred players in an attempt to observe whether there was an increased risk of injury on joining a new club. No statistically significant or practically meaningful differences in rates across seasons were observed. In the players' first season the incidence of injury overall ( 8.6 vs. 7.4 per 1000hours play) and in match play were nevertheless higher (40.4 vs. 32.5 per 1000hours play) despite a lower exposure time to competition (42 
vs. 47 hours). Reductions (trivial to small effect size differences) in mean layoff time per injury and the number of matches missed through injury also occurred in the player's second season. Despite these higher rates of injury overall and in competition and larger injury burden, these results can be interpreted positively as they demonstrate that newly transferred players were not at a substantially greater risk of sustaining injury or missing more matches through injury in their first season in a new club environment. Positively rates of non-contact type injuries such as muscle strains and joint sprains were comparable across seasons. In contrast, a practically meaningful greater risk of incurring a contact-type injury (2.9 vs. 1.5 per 1000 hours play) was observed in the player's first season which can partly explain the discrepancies in overall and match injury rates across seasons. Additional work to determine the causes behind the players sustaining more contact-type lesions is warranted.

Overall, these findings imply that the newly transferred players 'coped' with their new club environment and any potential changes in training load and methodology, styles of play and tactics. One reasonable explanation might be the combination of individualising player recommendations and programs guided via player screening (strength, movement and medical history consideration), injury prevention protocols (exercise based), daily monitoring (using key identified subjective and objective markers) and recovery strategies (cold water treatment, compression garments, nutrition) in place at the club over the entire study period (McCall et al., 2013; Nedelec et al., 2014). Another reason might be the history of previous injuries incurred by transferred players in their former club, which are clearly recognised as predisposing elite players to further injury (Hagglund, Walden \& Ekstrand, 2006). Research is warranted to investigate recruitment policies in the present club. For example, did recruitment and coaching staff account for injuries previously sustained in potential transfer targets (e.g., through mediabased epidemiological information) thereby identifying players with historically low injury rates?

In newly transferred players, injury incidences in their first and second seasons did not differ statistically compared to rates observed in players already playing at the club with a trend for lower values overall and in training. A practically meaningful trend for lower values for training rates in transferred players' first and second seasons was observed. Lower rates (albeit lacking practical meaningfulness) of injuries classed into non-contact, sprain, strain, overuse and categories also occurred. The aforementioned pre-season screening processes and prevention, recovery and monitoring protocols are again reasonable explanations for these findings. Another reason may be that the club's coaching staff operated squad rotation strategies in an attempt to prevent over-exposure in new players, maintain freshness and reduce the potential risk of injury in competition. Indeed, research previously conducted in the present club (Carling et al., 2015) showed that despite high availability rates for selection (>90\%), players considered first-team starters only participated in $>70 \%$ of all games over the course of a season. Supporting this point, match-play participation time for newly transferred players in their first season was lower than that observed in current squad members (42 versus 46 hours) suggesting that the former did not suffer from over-exposure. 
A clear limitation of this study was that the findings are derived from players belonging to a single elite football club and the patterns observed are a reflection of this particular cohort. Sample size strongly influences the statistical power needed to detect real and significant effects in epidemiological investigations. Here, 68 injury cases were reported in 25 individual players in their first-season while 200 injured participants are necessary to detect small-to-moderate associations, (Bahr \& Holmes, 2003). This preliminary case study work should therefore be extended to include a larger and wider sample of clubs and included as part of a multivariate approach to injury surveillance. In contrast, its strengths were the 8-season span and prospective data collection methodology the latter respecting international recommended injury recording systems thereby allowing the present data to be compared with future research findings.

In summary, the present findings on the extent of the injury problem in players newly transferred to a professional football club can generally be considered positive and lend weight to effective squad rotation strategies, the use of individual player injury programs and recovery strategies based on regular individual player screening and monitoring as essential components of the elite team program.

\section{References}

Bahr, R., \& Holme, I. (2003). Risk factors for sports injuries - A methodological approach. British Journal of Sports Medicine, 37, 384-392.

Batterham, A.M., \& Hopkins, W.G. (2006). Making meaningful inferences about magnitudes. International Journal of Sports Physiology \& Performance, 1, 50-57.

Carling, C., McCall, A., Le Gall, F., \& Dupont, G. (2015). What is the extent of exposure to periods of match congestion in professional soccer players? Journal of Sports Sciences, 33, 2116-2124.

Carling, C., McCall, A., Le Gall, F., \& Dupont, G. (2016). The impact of short periods of match congestion on injury risk and patterns in an elite football club. British Journal of Sports Medicine, 50, 764-768.

Eirale, C., Tol, J. L., Farooq, A., Smiley, F., \& Chalabi, H. (2013). Low injury rate strongly correlates with team success in Qatari professional football. British Journal of Sports Medicine, 47, 807-808.

Fuller, C. W., Ekstrand, J., Junge, A., Andersen, T. E., Bahr, R., Dvorak, J., ... Meeuwisse, W.H. (2006). Consensus statement on injury definitions and data collection procedures in studies of football (soccer) injuries. Clinical Journal of Sport Medicine, 16, 97-106.

Hagglund, M., Walden, M., Bahr, R., \& Ekstrand, J. (2005). Methods for epidemiological study of injuries to professional football players: Developing the UEFA model. British Journal of Sports Medicine, 39, 340-346.

Hägglund, M., Waldén, M., \& Ekstrand J. (2006). Previous injury as a risk factor for injury in elite football: a prospective study over two consecutive seasons. British Journal of Sports Medicine, 40, $767-$ 772. 
Hägglund, M., Waldén, M., Magnusson, H., Kristenson, K., Bengtsson, H., \& Ekstrand, J. (2013). Injuries affect team performance negatively in professional football: An 11-year follow-up of the UEFA Champions League injury study. British Journal of Sports Medicine, 47, 738-742.

Hayen A, \& Finch, C. (2010). Statistics used in effect studies. In E. Verhagen \& W. van Mechelen (Eds), Sports Injury Research pp. 181-193, New York: Oxford University Press.

Hopkins W.G (2006). A Spreadsheet for Combining Outcomes from Several Subject Groups. Sportscience 10, 51-53.

Hopkins, W. (2010). Statistics used in descriptive studies. In E. Verhagen \& W. van Mechelen (Eds), Sports Injury Research pp. 69-81, New York: Oxford University Press.Kristenson, K., Waldén, M., Ekstrand, J., \& Hägglund, M. (2013). Lower injury rates for newcomers to professional soccer: a prospective cohort study over 9 consecutive seasons. American Journal of Sports Medicine, 41, 14191425 .

Liu, X.F., Liu, Y., Lu, X., Wang Q., Wang, T. (2016). The Anatomy of the Global Football Player Transfer Network: Club Functionalities versus Network Properties. PLoS ONE 11(6), e0156504.

McCall, A., Carling, C., Nedelec, M., Davison, M., Le Gall, F., Berthoin, S., \& Dupont, G. (2014). Risk factors, testing and preventative strategies for non-contact injuries in professional football: current perceptions and practices of 44 teams from various premier leagues. British Journal of Sports Medicine, 48, 1352-1357.

Nédélec, M., McCall, A., Carling, C., Legall, F., Berthoin S., Dupont, G. (2013). Recovery in soccer: part ii-recovery strategies. Sports Medicine, 43, 9-22.

Stege J.P., Stubbe J.H., Verhagen EA., van Mechelen W. (2011). Risk factors for injuries in male professional soccer: a systematic review. British Journal of Sports Medicine, 45, 375-376.

Winter, E. M., \& Maughan, R. J. (2009). Requirements for ethics approvals. Journal of Sports Sciences, $27,985$. 
Table 1 Total exposure time in newly transferred players versus current first-team players across seasons in a professional football club.

2

\begin{tabular}{lccc}
\hline Exposure time (hours) & \multicolumn{2}{c}{ Transferred players } & Current players \\
& $1^{\text {st }}$ season $(\mathrm{n}=25)$ & $2^{\text {nd }}$ season $(\mathrm{n}=25)$ & $(\mathrm{n}=134)$ \\
\hline Total & 7879 & 8600 & 46499 \\
Training & 6829 & 7432 & 40300 \\
Matches & 1050 & 1168 & 6199 \\
\hline
\end{tabular}

3

4

Table 2 Incidences and patterns of injury in newly transferred players versus current first-team players across seasons in a professional football club.

\begin{tabular}{|c|c|c|c|c|c|c|c|c|c|c|c|c|}
\hline \multirow[b]{3}{*}{ Categories } & \multicolumn{3}{|c|}{ Incidence of injury } & \multirow{2}{*}{\multicolumn{2}{|c|}{$\begin{array}{l}\text { Transferred players } \\
1^{\text {st }} \mathrm{vs} 2^{\text {nd }} \text { seasons }\end{array}$}} & \multirow[b]{3}{*}{$\begin{array}{l}\text { Inference/ } \\
\text { \%Likelihood }\end{array}$} & \multicolumn{6}{|c|}{ Transferred vs. current players } \\
\hline & \multicolumn{2}{|c|}{ Transferred players } & \multirow{2}{*}{$\begin{array}{l}\text { Current players } \\
\qquad(\mathrm{n}=134)\end{array}$} & & & & \multirow[b]{2}{*}{ IRR $(95 \% \mathrm{CI})$} & \multicolumn{2}{|c|}{$1^{\text {st }}$ season } & \multicolumn{3}{|c|}{$2^{\text {nd }}$ season } \\
\hline & $1^{\text {st }}$ season $(\mathrm{n}=25)$ & $2^{\text {nd }}$ season $(n=25)$ & & IRR $(95 \% \mathrm{CI})$ & $\begin{array}{c}\mathrm{p} \\
\text { value }\end{array}$ & & & $\begin{array}{c}\mathrm{p} \\
\text { value }\end{array}$ & $\begin{array}{l}\text { Inference/ } \\
\text { \%Likelihood }\end{array}$ & $\begin{array}{l}\text { IRR } \\
(95 \% \mathrm{CI})\end{array}$ & $\begin{array}{l}\mathrm{p} \\
\text { value }\end{array}$ & $\begin{array}{l}\text { Inference/ } \\
\text { \%Likelihood }\end{array}$ \\
\hline Overall & $8.6(6.6-10.7)$ & $7.4(5.6-9.2)$ & $9.0(8.1-9.9)$ & $1.2(0.8-1.6)$ & 0.39 & $\begin{array}{l}\text { Possibly harmful } \\
7 / 32 / 60\end{array}$ & $1.0(0.7-1.2)$ & 0.73 & Unclear 33/54/13 & $0.8(0.6-1.1)$ & 0.14 & $\begin{array}{l}\text { Possibly beneficial } \\
75 / 24 / 1\end{array}$ \\
\hline Training & $2.8(1.5-4.1)$ & $3.1(1.8-4.4)$ & $4.3(3.7-4.9)$ & $0.9(0.5-1.7)$ & 0.74 & Unclear 50/23/27 & $0.7(0.4-1.0)$ & 0.08 & $\begin{array}{l}\text { Likely beneficial } \\
89 / 9 / 2\end{array}$ & $0.7(0.5-1.1)$ & 0.14 & $\begin{array}{l}\text { Likely beneficial } \\
82 / 14 / 4\end{array}$ \\
\hline Match & $40.4(27.6-53.2)$ & $32.5(22.2-42.9)$ & $37.3(32.5-42.1)$ & $1.2(0.8-1.9)$ & 0.32 & $\begin{array}{l}\text { Possibly harmful } \\
9 / 23 / 68\end{array}$ & $1.1(0.8-1.5)$ & 0.62 & $\begin{array}{l}\text { Possibly harmful } \\
15 / 40 / 45\end{array}$ & $0.9(0.6-1.2)$ & 0.43 & Unclear 57/34/9 \\
\hline Non-contact & $5.7(4.0-7.4)$ & $5.6(4.0-7.2)$ & $6.2(5.5-6.9)$ & $1.0(0.7-1.5)$ & 0.93 & Unclear 9/37/34 & $0.9(0.7-1.3)$ & 0.60 & Unclear 45/42/13 & $0.9(0.7-1.2)$ & 0.51 & Unclear 49/41/10 \\
\hline Contact & $2.9(1.7-4.1)$ & $1.9(1.0-2.8)$ & $2.8(2.3-3.3)$ & $1.5(0.8-2.9)$ & 0.19 & $\begin{array}{l}\text { Likely harmful } \\
6 / 12 / 82\end{array}$ & $1.0(0.7-1.6)$ & 0.88 & Unclear 28/33/39 & $0.7(0.4-1.1)$ & 0.14 & $\begin{array}{l}\text { Likely beneficial } \\
85 / 12 / 3\end{array}$ \\
\hline Re-injury & $1.3(0.5-2.1)$ & $1.0(0.3-1.7)$ & $1.3(1.0-1.6)$ & $1.3(0.5-3.2)$ & 0.57 & Unclear 26/13/61 & $1.0(0.5-1.9)$ & 0.98 & Unclear 38/23/39 & $0.8(0.4-1.6)$ & 0.46 & Unclear 63/15/21 \\
\hline Strains & $3.4(2.1-4.7)$ & $3.5(2.3-4.7)$ & $3.7(3.1-4.3)$ & $1.0(0.6-1.6)$ & 0.91 & Unclear 36/37/27 & $0.9(0.6-1.4)$ & 0.32 & Unclear $46 / 34 / 20$ & $1.0(0.6-1.4)$ & 0.78 & Unclear 40/39/21 \\
\hline Sprains & $1.3(0.5-2.1)$ & $1.3(0.5-2.0)$ & $1.4(1.1-1.7)$ & $1.0(0.4-2.4)$ & 0.98 & Unclear $42 / 16 / 42$ & $0.9(0.5-1.8)$ & 0.68 & Unclear $47 / 21 / 32$ & $0.9(0.5-1.8)$ & 0.82 & Unclear 47/22/32 \\
\hline Overuse & $0.5(0.0-1.0)$ & $0.6(0.1-1.1)$ & $1.0(0.7-1.3)$ & $1.1(0.3-4.3)$ & 0.97 & Unclear 53/7/40 & $0.5(0.2-1.4)$ & 0.18 & Unclear 76/7/17 & $0.5(0.2-1.4)$ & 0.18 & Unclear 74/10/16 \\
\hline
\end{tabular}

Injury incidences and Incidence Rate Ratios (IRR) are presented with 95\% Confidence Intervals (95\%CI).

$\%$ likelihood: effect is beneficial/trivial/harmful 